\title{
Synergistic Antioxidant Activity and Chemical Composition of Essential Oils From Thymus fontanesii, Artemisia herba- alba and Rosmarinus officinalis
}

\author{
Fatima Benyoucef ${ }^{1}$, Mohammed El Amine Dib ${ }^{2 *}$, Zoheir Arrar ${ }^{1}$, Jean Costa ${ }^{3}$, Alain Muselli ${ }^{3}$ \\ 1'Faculté des Sciences, Département de Chimie, Université de Tlemcen, Laboratoire (COSNA), BP 119, 13000 Tlemcen, Algeria \\ ${ }^{2}$ Laboratoire des Substances Naturelles et Bioactives (LASNABIO), Université de Tlemcen, BP 119, 13000, Algérie \\ ${ }^{3}$ UMR CNRS 6134, Campus Grimaldi, Université de Corse, Laboratoire CPN, BP 52, 20250 Corte, France
}

Corresponding Author: Mohammed El Amine Dib, PhD, Professor, Laboratoire des Substances Naturelles et Bioactives (LASNABIO), Université de Tlemcen, BP 119, 13000, Algérie. Tel : +21-3554378192,Email : a_dibdz@yahoo.fr

Received August 4, 2018; Accepted November 8, 2018; Online Published December 20, 2018

\begin{abstract}
Introduction: Oxidative stress is involved in many pathological mechanisms especially those due to aging, such as cancer, Parkinson's and Alzheimer's disease. Essential oils are known for their biological properties, especially as anti-nociceptive, anticancer, antiviral and antioxidative. The main objective of this study was to study the antioxidant activity of essential oils from Thymus fontanesii, Artemisia herba-alba and Rosmarinus officinalis, individually and in combinations.

Materials and Methods: Essential oils of plants aerial parts were analyzed by gas chromatography (GC) and gas chromatography-mass spectrometry (GC/MS). The antioxidant properties were evaluated using two different methods, $\alpha, \alpha$-diphenyl- $\beta$-picrylhydrazyl (DPPH) radical scavenging activity and ferric reducing antioxidant power (FRAP).

Results: The essential oil of $T$. fontanesii was principally characterized by phenolic compounds represented by thymol $(76.6 \%)$ and $p$-cymene (7.4\%). The constituents identified from A. herba-alba essential oil were principally represented by camphor (32.3\%) and chrysanthenone (25.6\%). While, R. officinalis essential oil was characterized by 1,8-cineole (18.3\%), camphene (15.4\%) and $\alpha$-pinene (12.8\%). T. fontanesii essential oil indicated the significantly highest activity in quenching of DPPH radical, followed by $R$. officinalis and $A$. herba-alba essential oils with IC $\mathrm{C}_{50}$ of 13.7 , 24.5 and $79.4 \mathrm{mg} / \mathrm{L}$, respectively. The combination of $T$. fontanesii, A. herba-alba and $R$. officinalis essential oils showed the greatest antioxidant activity with an $\mathrm{IC}_{50}$ of $2.6 \mathrm{mg} / \mathrm{L}$ almost equal to the synthetic antioxidant butylated hydroxytoluene (BHT).

Conclusions: The essential oils blend presented high antioxidant activity compared to individual oils. These findings provide a new source of antioxidant that can be used as a natural food preservative and alternative to chemical synthetic preservatives.

Keywords: Essential Oils, Antioxidant Activities, Synergistic Effects

Citation: Benyoucef F, El Amine Dib M, Arrar Z, Costa J, Muselli A. Synergistic antioxidant activity and chemical composition of essential oils from Thymus fontanesii, Artemisia herba-alba and Rosmarinus officinalis. J Appl Biotechnol Rep. 2018;5(4):151-156. doi:10.29252/JABR.05.04.03.
\end{abstract}

\section{Introduction}

In an attempt to preserve human health and avoid autooxidation affecting both the sensory and nutritional quality of foods, synthetic preservatives such as butylated hydroxytoluene (BHT) and butylated hydroxyanisole were introduced in the food industry. However, with the current resurgence of interest in the application of safe organic materials instead of synthetic materials, which are suspected for their carcinogenicity, essential oils are increasingly sought as natural alternatives. ${ }^{1,2}$ Essential oils represent a "green" alternative in the nutritional and pharmaceutical fields due to their incredible biological properties. ${ }^{3,4}$ Essential oils are not only used in monotherapy but also have been used in combinations for many years. ${ }^{5}$ They are used to act synergistically to further enhance their effects. The possible synergistic effect produced by the combination of plant essential oils was referred as an efficient strategy to inhibit or reduce the natural oxidation process of foods. ${ }^{6}$ The combination of essential oils with antioxidant effects approach may lead to new naturel preservatives. A Few research have studied the synergistic effect of plant essential oils. Grosso et al suggested that the combination of thymol, carvacrol and thymoquinone in the volatile oil of Satureja montana may be responsible for the increase in antioxidant activity, using the DPPH (2,2-diphenyl-1-picrylhydrazyl) radical scavenging and rancimat methods. ${ }^{7}$ Thymus fontanesii and Rosmarinus officinalis belong to the Lamiaceae family. While, Artemisia herba-alba belongs to the Asteraceae family. These species are especially known for their important biological properties such as antibacterial and antioxidant activities. To our knowledge, no study has investigated the antioxidant properties of $T$. fontanesii, $A$. herba-alba and $R$. officinalis essential oils blend. The purpose of the present study was $(i)$

Copyright (C) 2018 The Author(s). This is an open-access article distributed under the terms of the Creative Commons Attribution License (http:// creativecommons.org/licenses/by/4.0), which permits unrestricted use, distribution, and reproduction in any medium, provided the original work is properly cited. 
to determine the chemical composition of the essential oil of these plants, (ii) to evaluate the antioxidant power of each essential oil by DPPH and FRAP assays and (iii) to investigate the possible synergistic impacts of the combination of three essential oils.

\section{Materials and Methods}

Plant Material and Extraction of the Oils

Aerial parts of T. fontanesii, A. herba-alba and R. officinalis were collected at the flowering stage on May to June, 2017. The plant materials were botanically identified by the Laboratory of Ecology and Ecosystem Management of the University of Tlemcen, Algeria. Voucher specimens were deposited with the Herbarium of the University of Tlemcen. T. fontanesii (T.on.04/2018), A. herba-alba (A.h.a.05.2018) and $R$. officinalis (R.of 04/2018).

Essential oils were obtained from fresh material (300-400 g) by hydrodistillation for 5 hours using a Clevenger-type apparatus with yields (w/w) of $3.7 \%$ for $T$. fontanesii, $0,7 \%$ for A. herba-alba and $0.4 \%$ for R. officinalis.

\section{Gas Chromatography}

Gas chromatography (GC) analyses were carried out using a Perkin Elmer Clarus 600 GC apparatus (Walhton, MA, USA) equipped with a single injector and two flame ionization detectors (FIDs). The apparatus was used for simultaneous sampling of 2 fused-silica capillary columns $(60 \mathrm{~m} \times 0.22$ $\mathrm{mm}$, film thickness $0.25 \mu \mathrm{m}$ ) with different stationary phases: Rtx-1 (polydimethylsiloxane) and Rtx-Wax (polyethylene glycol). Temperature was programed 60 to $230^{\circ} \mathrm{C}$ at $2^{\circ} \mathrm{C} \mathrm{min}{ }^{-1}$ and then held isothermal $230^{\circ} \mathrm{C}$ (30 minutes). Carrier gas was hydrogen $\left(0.7 \mathrm{~mL} \mathrm{~min}^{-1}\right)$. Injector and detector temperatures were held at $280^{\circ} \mathrm{C}$. Split injection was conducted with a split ratio of 1:80. Injected volume was $0.1 \mu \mathrm{L}$.

\section{Gas Chromatography-Mass Spectrometry (GC/MS)}

The oils and the fractions obtained by GC were investigated using a Perkin Elmer TurboMass quadrupole analyzer, directly coupled with a Perkin Elmer Autosystem XL equipped with 2 fused-silica capillary columns $(60 \mathrm{~m} \times 0.22 \mathrm{~mm}$, film thickness $0.25 \mu \mathrm{m}), \mathrm{Rtx}^{-1}$ (polydimethylsiloxane) and RtxWax (polyethylene glycol). Other GC conditions were the same as described above. Ion source temperature was $150^{\circ} \mathrm{C}$ and energy ionization $70 \mathrm{eV}$; electron ionization mass spectra were acquired with a mass range of 35-350 Da and scan mass of $1 \mathrm{~s}$. Oil injected volume was $0.1 \mu \mathrm{L}$ and fraction injected volume was $0.2 \mu \mathrm{L}$.

\section{Component Identification}

The identification of each compound was carried out by comparison: $(i)$ retention indices calculated respectively on polar and apolar columns with those of standard compounds of the laboratory library ${ }^{8,9}$ or those reported in the literature; (ii) mass spectra (electronic impact) with those of standard compounds or those present in computerized banks. ${ }^{10,11}$

Determination of Antioxidant Activity of Essential Oils DPPH Free Radical Scavenging Assay
The free radical-scavenging activity of essential oils and combinations were measured using $\mathrm{DPPH}$, as described in the literature. ${ }^{12}$ At first, $500 \mathrm{mg} / \mathrm{L}$ of each essential oil stock solution was prepared. A series of dilution with varying concentrations $(0.1$ to $100 \mathrm{mg} / \mathrm{L})$ was prepared by dissolving various masses of essential oil in ethanol. In regards to essential oils blends, a ratio of 1: 1 by volume was mixed for each combination and a series of concentrations ranging from 0.1 to $50 \mathrm{mg} / \mathrm{L}$ were prepared. After on, $100 \mu \mathrm{L}$ of each concentration was then mixed with $25 \mu \mathrm{L}$ of $0.5 \mathrm{mM}$ DPPH. After a 30 minutes incubation period at room temperature, the absorbance was measured at $517 \mathrm{~nm}$ using spectrophotometer. Ascorbic acid was used as standard and DPPH mixture without any sample served as blank. Inhibition of free radical DPPH in percent (I\%) was calculated as follows:

$\mathrm{I} \%=\left[\frac{\mathrm{A}_{\text {blank }}-\mathrm{A}_{\text {sample }}}{\mathrm{A}_{\text {blank }}}\right] \times 100 \%$

As presented above, $A_{\text {blank }}$ is the absorbance of the control reaction (without oils), and $\mathrm{A}_{\text {sample }}$ was the absorbance in the presence of essential oils. From the obtained RSC values, the $\mathrm{IC}_{50}$ values, which represented the concentrations of the extracts caused 50\% neutralization, were determined by linear regression analysis.

\section{Ferric-Reducing Antioxidant Power Assay}

The ferric reducing antioxidant power (FRAP) of the essential oils was tested as described earlier ${ }^{13}$. Different concentrations of the essential oils dissolved in ethanol (80\%) were mixed with $2.5 \mathrm{~mL}$ of phosphate buffer $(\mathrm{pH}=6.6)$ and $2.5 \mathrm{~mL}$ of potassium ferricyanide. Later, the mixture was incubated at $50^{\circ} \mathrm{C}$ for 20 minutes, and then trichloroacetic acid $(10 \%$, $1,25 \mathrm{~mL}$ ) was added. After shaking the mixture vigorously, it was mixed with distilled water $(5 \mathrm{~mL})$ and ferric chloride $(0.1 \%, 5 \mathrm{~mL})$. After 30 minutes of incubation, absorbance was read at $700 \mathrm{~nm}$ against ethanol (80\%) as blank. Analyses were performed in triplicate. Increased absorbance of the reaction meant increased reducing power and compared to the synthetic antioxidant BHT as reference.

\section{Statistical Analysis}

The data are presented as the means \pm standard deviations from the three replicates. Calculations were performed using the SAS v. 9.1.3 program.

\section{Results}

Chemical Composition of Essential Oils

GC-FID and GC-MS analysis of T. fontanesii, A. herbaalba and R. officinalis oils accounted for 99.3\%, 93.4\% and $91.1 \%$ of oils, respectively and allowed the identification of 31, 20 and 19 components, respectively (Table 1). All components were identified by comparison of their mass spectrum and retention indices with those of our laboratoryproduced "Arômes" library (Table 1). The essential oil of T. fontanesii obtained from the aerial parts was dominated principally by monoterpenoid phenol (84.7\%). The main components were thymol (76.6\%) and p-cymene (7.4\%). 
Table 1. Chemical Composition of the Essential Oils

\begin{tabular}{|c|c|c|c|c|c|c|c|c|}
\hline \multirow{2}{*}{$\mathrm{No}^{\mathrm{a}}$} & \multirow{2}{*}{ Compounds } & \multirow{2}{*}{$\mid \mathbf{R I}_{\mathrm{a}}{ }^{\mathrm{b}}$} & \multirow{2}{*}{$\mathbf{R I}_{\mathrm{a}}^{\mathrm{c}}$} & \multirow{2}{*}{$\mathbf{R I}_{\mathrm{p}}{ }^{\mathrm{d}}$} & \multicolumn{3}{|c|}{ Essential oils } & \multirow{2}{*}{ Identification } \\
\hline & & & & & T. fontanesii & A. herba-alba & R. officinalis & \\
\hline 1 & $\alpha$-Thujene & 932 & 924 & 1028 & 0.2 & - & - & RI. MS \\
\hline 2 & $\alpha$-Pinene & 936 & 931 & 1028 & 0.9 & 0.3 & 12.8 & RI. MS \\
\hline 3 & Camphene & 950 & 945 & 1071 & 0.2 & 10.3 & 0.2 & RI. MS \\
\hline 4 & Oct-1-en-3-ol & 962 & 962 & 1441 & 0.5 & - & - & RI. MS \\
\hline 6 & Myrcenes & 987 & 982 & 1160 & 2.1 & - & 0.1 & RI. MS \\
\hline 7 & $\alpha$-Phellandrene & 1002 & 999 & 1161 & 0.2 & - & 0.2 & RI. MS \\
\hline 8 & 3-Carene & 1005 & 1006 & 1149 & 0.1 & - & - & RI. MS \\
\hline 9 & $\alpha$-Terpinene & 1008 & 1011 & 1270 & 1.7 & - & - & RI. MS \\
\hline 10 & p-Cymene & 1015 & 1015 & 1270 & 7.4 & - & 1.5 & RI. MS \\
\hline 11 & 1.8-Cineole & 1024 & 1021 & 1211 & - & 8.4 & 18.3 & RI. MS \\
\hline 12 & (Z)- $\beta$-Ocimene & 1029 & 1022 & 1234 & 0.6 & - & - & RI. MS \\
\hline 13 & Limonene & 1039 & 1031 & 1967 & - & - & 4.2 & RI. MS \\
\hline 14 & $\gamma$-Terpinene & 1051 & 1050 & 1245 & 2.3 & 0.2 & 3.8 & RI. MS \\
\hline 15 & trans-Sabinene hydrate & 1051 & 1054 & 1445 & 0.1 & 0.1 & - & RI. MS \\
\hline 16 & Terpinolene & 1082 & 1079 & 1281 & 0.2 & - & - & RI. MS \\
\hline 17 & Linalool & 1083 & 1085 & 1538 & 1.7 & 0.5 & - & RI. MS \\
\hline 18 & Chrysanthenone & 1110 & 1106 & 1504 & - & 25.6 & - & RI. MS \\
\hline 19 & Camphor & 1123 & 1124 & 1506 & 0.1 & 32.3 & - & RI. MS \\
\hline 20 & $\alpha$-pinocarvone & 1135 & 1139 & 1632 & - & - & 1.4 & RI. MS \\
\hline 21 & Camphre & 1144 & 1145 & 1532 & - & - & 15.4 & RI. MS \\
\hline 22 & Borneol & 1148 & 1150 & 1688 & 0.3 & 4.5 & 12.7 & RI. MS \\
\hline 23 & Terpinen-4-ol & 1164 & 1162 & 1591 & 1.1 & 3.2 & 1.2 & RI. MS \\
\hline 26 & Carvone & 1214 & 1215 & 1278 & - & 3.5 & - & RI. MS \\
\hline 27 & Thymol & 1266 & 1263 & 2181 & 76.6 & - & - & RI. MS \\
\hline 28 & Carvacrol & 1278 & 1286 & 2193 & 0.6 & - & - & RI. MS \\
\hline 29 & Bornyl acetate & 1285 & 1280 & 1600 & - & - & 2.6 & RI. MS \\
\hline 30 & Eugenol & 1330 & 1329 & 2164 & 0.1 & - & - & RI. MS \\
\hline 31 & cis-Carvyl acetate & 1343 & 1345 & 1858 & 0.1 & - & - & RI. MS \\
\hline 32 & (E)- $\beta$-Caryophyllene & 1421 & 1416 & 1591 & 1.6 & - & 0.2 & RI. MS \\
\hline 33 & $(E)-\alpha$-Bergamotene & 1434 & 1435 & 1573 & Trace & 0.1 & - & RI. MS \\
\hline 34 & $\alpha$-Humulene & 1455 & 1448 & 1668 & 0.1 & 0.1 & 0.6 & RI. MS \\
\hline 35 & $\gamma$-Humulene & 1483 & 1480 & 1702 & Trace & 0.1 & - & RI. MS \\
\hline 36 & $\beta$-Bisabolene & 1503 & 1499 & 1721 & 0.1 & 0.2 & - & RI. MS \\
\hline 37 & $\delta$-Cadinene & 1520 & 1511 & 1760 & Trace & 0.5 & - & RI. MS \\
\hline 38 & (E)- $\alpha$-Bisabolne & 1530 & 1531 & 1755 & 0.1 & 0.3 & - & RI. MS \\
\hline 39 & Spathulenol & 1572 & 1560 & 2120 & - & 0.5 & - & RI. MS \\
\hline 40 & Globulol & 1589 & 1578 & 2067 & - & 0.6 & - & RI. MS \\
\hline 41 & Caryophyllene oxide & 1578 & 1567 & 1969 & 0.1 & - & 1.4 & RI. MS \\
\hline \multicolumn{3}{|c|}{ Total identification \% } & & & 99.3 & 93.4 & 91.1 & \\
\hline \multicolumn{3}{|c|}{ Monoterpene hydrocarbons \% } & & & 8.6 & 10.8 & 21.5 & \\
\hline \multicolumn{3}{|c|}{ Sesquiterpene hydrocarbons $\%$} & & & 1.9 & 1.3 & 0.8 & \\
\hline \multicolumn{3}{|c|}{ Oxygenated monoterpenes \% } & & & 3.6 & 80.2 & 65.9 & \\
\hline \multicolumn{3}{|c|}{ Oxygenated sesquiterpenes \% } & & & 0.1 & 1.1 & 1.4 & \\
\hline \multicolumn{3}{|c|}{ Non terpenic oxygenated compounds \% } & & & 0.5 & - & - & \\
\hline \multicolumn{3}{|c|}{ Monoterpenoid phenol. \% } & & & 84.7 & - & 1.5 & \\
\hline
\end{tabular}

a Order of elution is given on apolar column (Rtx-1).

${ }^{b}$ Retention indices of literature on the apolar column (IRla) reported from Konig. ${ }^{9}$

${ }^{c}$ Retention indices on the apolar Rtx-1 column (Rla).

${ }^{\mathrm{d}}$ Retention indices on the polar Rtx-Wax column (RIp).

e Identification mode; RI. retention indices; MS. mass spectrometry in electron impact mode. 
The constituents identified in the aerial parts of $R$. officinalis and $A$. herba-alba essential oils were principally oxygenated monoterpenes ( 80.2 and $65.9 \%$, respectively), followed by monoterpene hydrocarbons ( 10.8 and $21.5 \%$, respectively). The main components of $A$. herba-alba essential oil were camphor (32.3\%), chrysanthenone (25.6\%), 1,8-cineole (8.4\%) and borneol (4.5\%). While, R. officinalis essential oil was characterized by 1,8 -cineole $(18.3 \%)$, camphene $(15.4 \%)$, a-pinene (12.8\%), borneol (12.7\%), verbenone (12.7\%) and limonene $(4.2 \%)$ (Table 1$)$.

\section{DPPH Free Radical Scavenging Assay}

All essential oils were able to reduce the stable free radical $\mathrm{DPPH}$ to the yellow colored diphenylpicrylhydrazine. However, T. fontanesii presented the highest antioxidant effect based on DPPH test that had the lowest $\mathrm{IC}_{50}$ value of $13.7 \mathrm{mg} / \mathrm{L}$. While, A. herba-alba and R. officinalis essential oils appeared to be the least active oils with $\mathrm{IC}_{50}$ values of 24.5 and $79.4 \mathrm{mg} / \mathrm{L}$, respectively (Table 2 ).

Essential oils blends were very effective compared to the individual essential oils tested. The combination of $T$. fontanesii, A. herba-alba and R. officinalis essential oils showed a significant increase in antioxidant activity with an $\mathrm{IC}_{50}$ of $2.6 \mathrm{mg} / \mathrm{L}$, almost equal to the synthetic antioxidant used as reference $\left(\mathrm{IC}_{50}=2,3 \mathrm{mg} / \mathrm{L}\right.$ ) (Table 3 ). The combination of essential oils of $T$. fontanesii and $R$. officinalis showed also good antioxidant activity with an $\mathrm{IC}_{50}$ of $7.2 \mathrm{mg} / \mathrm{L}$. However, the essential oils blends of T. fontanesii and A. herba-alba, and $A$. herba-alba and $R$. officinalis had promising antioxidant activities with $\mathrm{IC}_{50}$ s of 23.9 and $39.2 \mathrm{mg} / \mathrm{L}$, but were still lower than the synthetic antioxidant (BHT) (Table 3).
Ferric Reducing Antioxidant Power Assay

FRAP assay is a widely used method that uses antioxidants as reductants in a redox-linked colorimetric reaction, wherein $\mathrm{Fe}^{3+}$ is reduced to $\mathrm{Fe}^{2+}$. Figure 1 depicts the reducing power of individual and blend essential oils. All oils showed the presence the reductive effects, which increased with an increase in concentration. However, the combination of $T$. fontanesii, A. herba-alba and R. officinalis essential oils has been as effective on reducing power, compared to the synthetic antioxidant BHT. The individual and blends essential oils displayed chelating effects on ferrous ions, suggesting that it can sequestrate Fe-ions or reduce the concentration of metal.

\section{Discussion}

As a result, T. fontanesii presented the highest antioxidant effect, probably related to its chemical profile, especially the relatively high percentage of phenolic compounds. Thymol and p-cymene are the most frequently occurring constituents of essential oils obtained from thyme species, with many biological activities. They also act as antioxidants (free radical scavenger, anti-lipid peroxidative agent, etc). ${ }^{14-16}$ The moderated antioxidant activity obtained from $R$. officinalis essential oil could be the consequence of appreciable content of camphene, borneol, verbenone, 1,8-cineole and $\alpha$-pinene, that represented more than $71 \%$ of the total oil. The lowest antioxidant activity was recorded for $A$. herba-alba oil where chrysanthenone, camphor, camphene and 1,8-cineole represented more than $66.3 \%$ of the total oil. The antioxidant activity obtained from $A$. herba-alba and $R$. officinalis essential oils could be the consequence of appreciable contents of oxygenated monoterpenes ( 80.2 and $65.9 \%$, respectively)

Table 2. 2.2-Diphenyl-1-picrylhydrazil Radical Scavenging Activities (\%)

\begin{tabular}{|c|c|c|c|c|c|c|c|c|}
\hline \multicolumn{2}{|l|}{ Samples } & \multicolumn{6}{|c|}{ Antioxidant Activity } & \multirow{3}{*}{$\begin{array}{c}\mathbf{I C}_{\mathbf{5 0}} \\
13.7 \pm 1.1\end{array}$} \\
\hline \multirow{2}{*}{ Thymus fontanesii } & Concentration (mg/L) & 0.2 & 1 & 5 & 10 & 15 & 20 & \\
\hline & DPPH radical scavenging activity (\%) & 3.1 & 5.8 & 19.8 & 37.2 & 54.6 & 72.1 & \\
\hline \multirow{2}{*}{ Rosmarinus officinalis } & Concentration (mg/L) & 5 & 10 & 15 & 20 & 25 & 30 & \multirow{2}{*}{$24.5 \pm 2.1$} \\
\hline & DPPH radical scavenging activity $(\%)$ & 5.9 & 17.2 & 28.5 & 39.8 & 51.1 & 62.4 & \\
\hline \multirow{2}{*}{ Artemisia herba-alba } & Concentration (mg/L) & 10 & 20 & 30 & 50 & 70 & 90 & \multirow{2}{*}{$79.4 \pm 2.8$} \\
\hline & DPPH radical scavenging activity $(\%)$ & 10.1 & 15.8 & 21.6 & 38.8 & 44.6 & 50.4 & \\
\hline \multirow{2}{*}{ BHT } & Concentration (mg/L) & 0.2 & 0.5 & 1.0 & 2.0 & 3.0 & 4.0 & \multirow{2}{*}{$2.3 \pm 0.8$} \\
\hline & DPPH radical scavenging activity $(\%)$ & 28.4 & 35.6 & 47.9 & 71.9 & 85.1 & 86.6 & \\
\hline
\end{tabular}

Table 3. Antioxidant Activity of Essential Oils Blends

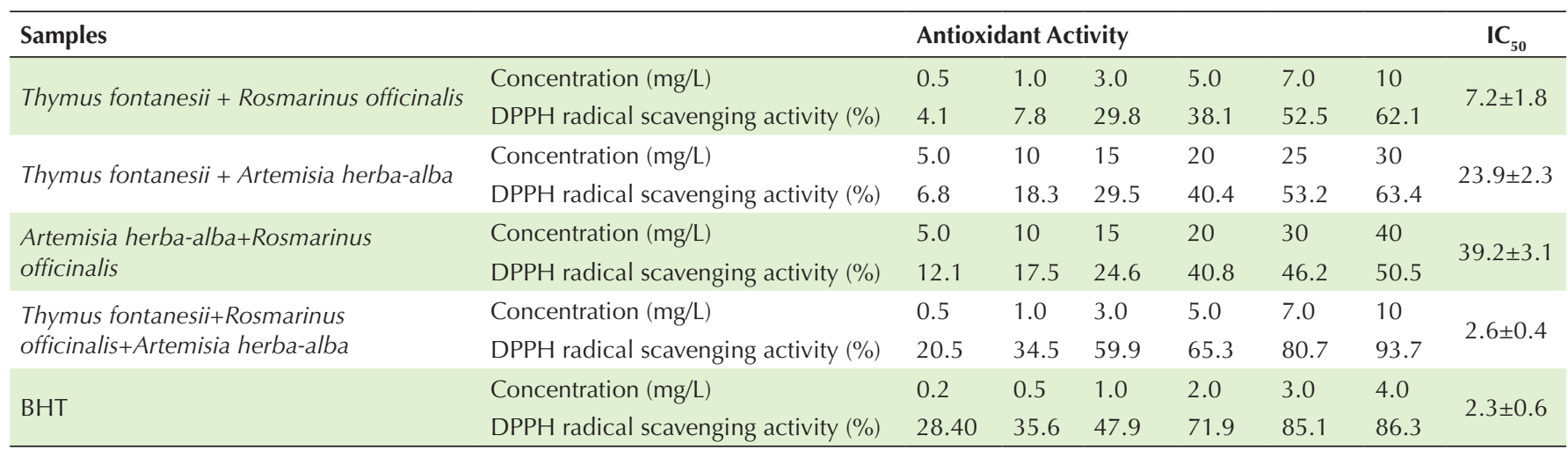




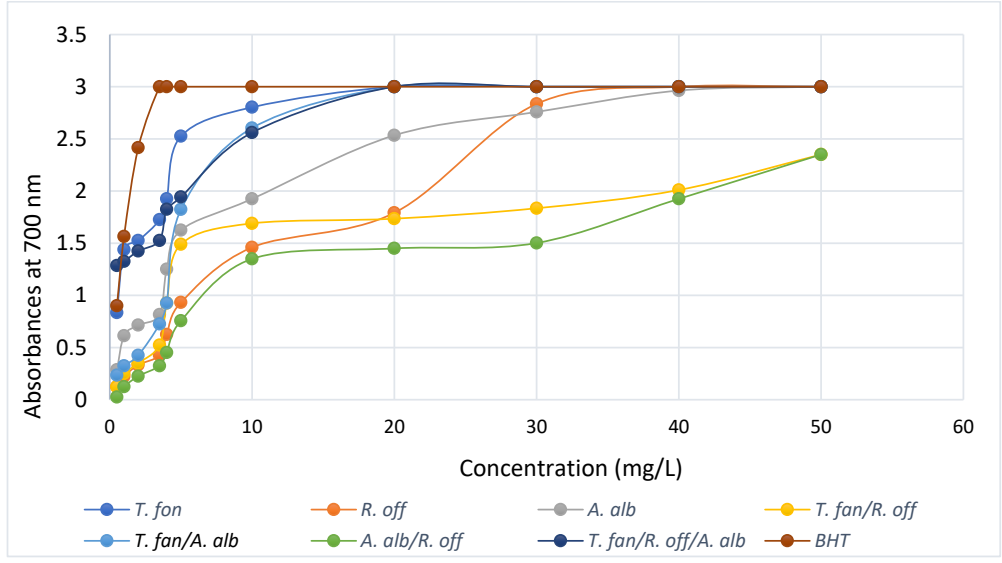

Figure 1. Ferric-Reducing Antioxidant Power Assay of Individual and Blend Essential Oils. Abbreviations: T. fan, Thymus fontanesii; R. off, Rosmarinus officinalis; A. alb, Artemisia herba-alba; BHT, butylated hydroxytoluene.

such as 1,8 cineole, camphor, chrysanthenone, verbenone and borneol. Indeed, Dawidowicz and Olszowy ${ }^{17}$ showed that camphor, one of the main components in essential oil of Salvia hispanica, has lower antioxidant activity. This is while, 1,8 cineole and borneol were naturally found in many aromatic plants and showed weak antioxidant activity. ${ }^{18,19}$ However, no information was found in the available literature about the biological activities of chrysanthenone and verbenone. Furthermore, monoterpene hydrocarbons are known to have noticeable antioxidant activities. ${ }^{20}$ The obtained findings showed that essential oil combinations applied a promising synergistic antioxidant effect by decreasing the half maximal inhibitory concentration. The strong synergistic effect was found by combining T. fontanesii, A. herba-alba and $R$. officinalis essential oils (Table 3 ). It seems that the association of thymol and p-cymene with 1,8-cineole, chrysanthenone, camphor, borneol and verbenone increases the antioxidant activity. T. fontanesii and $R$. officinalis essential oils blend also showed a good antioxidant capacity when compared to the value of the synthetic antioxidant. It is evident that this synergistic effect was found when thymol and p-cymene was paired with 1,8-cineole, borneol and verbenone. However, the blends of $T$. fontanesii and A. herba-alba, A. herba-alba and $R$. officinalis essential oils showed the lowest antioxidant activities. It seems that the combination of thymol and p-cymene with chrysanthenone, camphor and 1,8-cineole produce low antioxidant power. While, the absence of thymol and p-cymene of blend significantly decreases this power.

In conclusion, the results showed that essential oil of T. fontanesii is a good source of monoterpenoid phenols as thymol and p-cymene. The essential oil had the best antioxidant activity using DPPH and FRAP methods. While, R. officinalis and A. herba-alba essential oils rich with oxygenated monoterpenes showed the lowest antioxidant activities by means of highest $\mathrm{IC}_{50}$ values. On the other hand, blends of essential oils showed an increase of antioxidant capacity. It seems that the monoterpenoid phenols (i.e. thymol and p-cymene) associated with oxygenated monoterpenes such as 1.8-cineole, chrysanthenone, camphor, borneol and verbenone play a pivotal role in this activity and produce stronger synergistic effect. These results, therefore, represent a basis for further studies that could lead to the development of a new treatment based on the combination of these essential oils as natural antioxidant agents, both in food and pharmaceuticals fields.

\section{Authors' Contributions}

FB prepared the samples and carried out the experiments; MAD wrote the manuscript; $A Z$ conceived the original idea; AM performed the analyzes; JC supervised the project.

\section{Conflict of Interest Disclosures}

The authors declare they have no conflicts of interest.

\section{Ethical Approval}

Not applicable.

\section{References}

1. Namiki M. Antioxidants/antimutagens in food. Crit Rev Food Sci Nutr. 1990;29(4):273-300. doi:10.1080/10408399009527528.

2. Pokorny J. Natural antioxidants for food use. Trends Food Sci Technol. 1991;2:223-227. doi:10.1016/0924-2244(91)90695-F.

3. Papachristos DP, Karamanoli KI, Stamopoulos DC, MenkissogluSpiroudi $U$. The relationship between the chemical composition of three essential oils and their insecticidal activity against Acanthoscelides obtectus (Say). Pest Manag Sci. 2004;60(5):514520. doi:10.1002/ps.798.

4. Swamy MK, Sinniah UR. A comprehensive review on the phytochemical constituents and pharmacological activities of Pogostemon cablin Benth.: an aromatic medicinal plant of industrial importance. Molecules. 2015;20(5):8521-8547. doi:10.3390/molecules20058521.

5. Blumenthal M. Herbal medicines: expanded commission E monographs. Boston: Integrative Medicine Communications; 2000:419-423.

6. Bozin B, Mimica-Dukic N, Simin N, Anackov G. Characterization of the volatile composition of essential oils of some lamiaceae spices and the antimicrobial and antioxidant activities of the entire oils. J Agric Food Chem. 2006;54(5):1822-1828. doi:10.1021/ jf051922u.

7. Grosso C, Oliveira AC, Mainar AM, Urieta JS, Barroso JG, Palavra AM. Antioxidant activities of the supercritical and conventional Satureja montana extracts. J Food Sci. 2009;74(9):C713-717. doi:10.1111/j.1750-3841.2009.01376.x.

8. Jennings W, Shibamoto T. In: Jovanovich HB (ed). Qualitative analysis of flavour and fragrance volatiles by glass-capillary gas 
chromatography. New York: Academic Press; 1980.

9. Konig WA, Hochmuth DH, Joulain D. Terpenoids and related constituents of essential oils library of mass finder 2.1. Ed. Hamburg: Institute of Organic Chemistry; 2001.

10. McLafferty FW, Stauffer DB. The Wiley/NBS registry of mass spectral data. Ed, New York: Wiley-Interscience; 1988.

11. McLafferty FW, Stauffer DB. Wiley registry of mass spectral data. 6th ed. Mass Spectrometry Library Search System Bench-Top/PBM version 3.10d. Palisade: Newfield. 1994.

12. Hatami T, Emami SA, Miraghaee SS, Mojarrab M. Total phenolic contents and antioxidant activities of different extracts and fractions from the aerial parts of Artemisia biennis Willd. Iran J Pharm Res. 2014;13(2):551-558. doi:10.22037/ijpr.2014.1518.

13. Al-Qudah MA, Obeidat SM, Saleh AM, et al. Volatile components analysis, total phenolic, flavonoid contents, and antioxidant activity of Phlomis species collected from Jordan. Journal of Essential Oil Bearing Plants. 2018;21(3):583-599. doi:10.1080/0 972060X.2018.1489739.

14. Beena, Kumar D, Rawat DS. Synthesis and antioxidant activity of thymol and carvacrol based Schiff bases. Bioorg Med Chem Lett. 2013;23(3):641-645. doi:10.1016/j.bmcl.2012.12.001.

15. Mahmoud AL. Antifungal action and antiaflatoxigenic properties of some essential oil constituents. Lett Appl Microbiol.
1994;19(2):110-113. doi:10.1111/j.1472-765X.1994.tb00918.x.

16. Saad A, Fadli M, Bouaziz M, Benharref A, Mezrioui NE, Hassani L. Anticandidal activity of the essential oils of Thymus maroccanus and Thymus broussonetii and their synergism with amphotericin B and fluconazol. Phytomedicine. 2010;17(13):1057-1060. doi:10.1016/j.phymed.2010.03.020.

17. Dawidowicz AL, Olszowy M. Does antioxidant properties of the main component of essential oil reflect its antioxidant properties? The comparison of antioxidant properties of essential oils and their main components. Nat Prod Res. 2014;28(22):1952-1963. doi:10 $.1080 / 14786419.2014 .918121$.

18. Zengin H, Baysal AH. Antibacterial and antioxidant activity of essential oil terpenes against pathogenic and spoilage-forming bacteria and cell structure-activity relationships evaluated by SEM microscopy. Molecules. 2014;19(11):17773-17798. doi:10.3390/ molecules191117773.

19. Chen L, Su J, Li L, Li B, Li W. A new source of natural D-borneol and its characteristic. J Med Plant Res. 2011;5(15):3440-3447.

20. Elansary HO, Salem MZM, Ashmawy NA, Yacout MM. Chemical composition, antibacterial and antioxidant activities of leaves essential oils from Syzygium cumini L., Cupressus sempervirens L. and Lantana camara L. from Egypt. J Agric Sci. 2012;4(10):144152. doi:10.5539/jas.v4n10p144. 